Nonlinear Processes in Geophysics, 13, 9-22, 2006

SRef-ID: 1607-7946/npg/2006-13-9

European Geosciences Union

(C) 2006 Author(s). This work is licensed

under a Creative Commons License.

\title{
A quasi-normal scale elimination model of turbulence and its application to stably stratified flows
}

\author{
S. Sukoriansky ${ }^{1}$, B. Galperin ${ }^{2}$, and V. Perov ${ }^{3}$ \\ ${ }^{1}$ Department of Mechanical Engineering/Perlstone Center for Aeronautical Engineering Studies, Ben-Gurion University of \\ the Negev, Beer-Sheva 84105, Israel \\ ${ }^{2}$ College of Marine Science, University of South Florida, St. Petersburg, Florida 33701, USA \\ ${ }^{3}$ Department of Research and Development, Swedish Meteorological and Hydrological Institute, SE-601 76 Norrköping, \\ Sweden
}

Received: 9 September 2005 - Revised: 23 December 2005 - Accepted: 23 December 2005 - Published: 3 February 2006

Part of Special Issue "Turbulent transport in geosciences"

\begin{abstract}
Models of planetary, atmospheric and oceanic circulation involve eddy viscosity and eddy diffusivity, $K_{M}$ and $K_{H}$, that account for unresolved turbulent mixing and diffusion. The most sophisticated turbulent closure models used today for geophysical applications belong in the family of the Reynolds stress models. These models are formulated for the physical space variables; they consider a hierarchy of turbulent correlations and employ a rational way of its truncation. In the process, unknown correlations are related to the known ones via "closure assumptions" that are based upon physical plausibility, preservation of tensorial properties, and the principle of the invariant modeling according to which the constants in the closure relationships are universal. Although a great deal of progress has been achieved with Reynolds stress closure models over the years, there are still situations in which these models fail. The most difficult flows for the Reynolds stress modeling are those with anisotropy and waves because these processes are scaledependent and cannot be included in the closure assumptions that pertain to ensemble-averaged quantities. Here, we develop an alternative approach of deriving expressions for $K_{M}$ and $K_{H}$ using the spectral space representation and employing a self-consistent, quasi-normal scale elimination (QNSE) algorithm. More specifically, the QNSE procedure is based upon the quasi-Gaussian mapping of the velocity and temperature fields using the Langevin equations. Turbulence and waves are treated as one entity and the effect of the internal waves is easily identifiable. This model implies partial averaging and, thus, is scale-dependent; it allows one to easily introduce into consideration such parameters as the grid resolution, the degree of the anisotropy, and spectral characteristics, among others. Applied to tur-
\end{abstract}

Correspondence to: S. Sukoriansky

(semion@bgu.ac.il) bulent flows affected by anisotropy and waves, the method traces turbulence anisotropization and shows how the dispersion relationships for linear waves are modified by turbulence. In addition, one can derive the internal wave frequency shift and the threshold criterion of internal wave generation in the presence of turbulence. The spectral method enables one to derive analytically various one-dimensional and three-dimensional spectra that reflect the effects of waves and anisotropy. When averaging is extended to all scales, the method yields a Reynolds-averaged, Navier-Stokes equations based model (RANS). This RANS model shows that there exists a range of $R i$, approximately between 0.1 and 1 , in which turbulence undergoes remarkable anisotropization; the vertical mixing becomes suppressed while the horizontal mixing is enhanced. Although $K_{H}$ decreases at large $R i$ and tends to its molecular value, $K_{M}$ remains finite and larger than its molecular value. This behavior is attributable to the effect of internal waves that mix the momentum but do not mix a scalar. In the Reynolds stress models, this feature is not replicated; instead, all Reynolds stress models predict $K_{M} \rightarrow 0$ at some value of $R i \leq 1$ which varies from one model to another. The presented spectral model indicates that there is no a single-valued critical Richardson number $R i$ at which turbulence is fully suppressed by stable stratification. This result is in agreement with large volume of atmospheric, oceanic and laboratory data. The new spectral model has been implemented in the $K-\epsilon$ format and tested in simulations of the stably stratified atmospheric boundary layers. The results of these simulations are in good agreement with the data collected in BASE, SHEBA and CASES99 campaigns. Implementation of the QNSE-derived $K_{M}$ and $K_{H}$ in the high-resolution weather prediction system HIRLAM results in significant improvement of its predictive skills. 


\section{Introduction}

Turbulence is one of the principal unsolved problems in physics. Difficulties in the theory of turbulence stem from strong nonlinearity of the equations of motion. Various instabilities at large Reynolds number, $R e$, lead to excitation of secondary flows, such as vortices and waves, developing on different spatial and temporary scales. Nonlinear interactions between motions on various scales generate highly irregular, "stochastic" flow fields. Solutions exist only for simplest flows that are locally isotropic and depend on a single nondimensional parameter $R e$.

In the context of geophysical, planetary and astrophysical turbulence, turbulent flows are further complicated by such factors as spatial anisotropy and waves. On relatively small scales, the gravity force causes density stratification and emergence of internal gravity waves. On larger scales, the Coriolis force, caused by the planetary rotation, leads to quasi-two-dimensionalization of the flow and emergence of inertial waves. On yet larger, planetary scales, the variation of the Coriolis force with latitude, or the so-called $\beta$-effect, leads to the emergence of Rossby waves and flow zonation. Models of turbulence used to simulate all these flows must be capable of accounting for different effects on different scales but Reynolds averaging does not differentiate between scales lumping them all together. On the other hand, a spectral approach does account for scale-specific phenomena. This paper is concerned with the development of a spectral model for turbulent flows with stable density stratification.

The article is organized in the following fashion. Section 2 presents the basics of the quasi-normal scale elimination theory and explains the derivation of the scale-dependent, horizontal and vertical, eddy viscosities and eddy diffusivities. Section 3 shows some theoretical results that can be obtained from the model; they include the dispersion relation of internal waves in the presence of turbulence, the threshold of internal waves generation, and various one-dimensional spectra. Section 4 explains how RANS models can be derived from the spectral model while Sect. 5 compares the spectral model results with laboratory and observational data. Section 6 provides a brief description of the $K-\epsilon$ model that employs the vertical eddy viscosities and eddy diffusivities derived from the spectral model while Sect. 7 demonstrates that the use of these eddy viscosities and eddy diffusivities improves the predictive skills of a high-resolution, limited area, numerical weather forecast system HIRLAM. Finally, Sect. 8 provides a discussion and conclusions.

\section{Basics of the spectral theory of turbulent flows with stable stratification}

\subsection{Governing equations and their Fourier transform}

The spectral theory is developed for a fully threedimensional turbulent flow field with imposed vertical, stabilizing temperature gradient. The flow is governed by the mo- mentum, temperature and continuity equations in the Boussinesq approximation,

$$
\begin{aligned}
\frac{\partial \mathbf{u}}{\partial t}+(\mathbf{u} \cdot \nabla) \mathbf{u}-\alpha g T \hat{e}_{3} & =v_{0} \nabla^{2} \mathbf{u}-\frac{1}{\rho} \nabla P+\mathbf{f}^{\mathbf{0}}, \\
\frac{\partial T}{\partial t}+(\mathbf{u} \cdot \nabla) T+\frac{d \Theta}{d z} u_{3} & =\kappa_{0} \nabla^{2} T, \\
\nabla \cdot \mathbf{u} & =0
\end{aligned}
$$

where $P$ is the pressure, $\rho$ is the constant reference density, $\nu_{0}$ and $\kappa_{0}$ are the molecular viscosity and diffusivity, respectively, $\alpha$ is the thermal expansion coefficient, $g$ is the acceleration due to gravity directed downwards, $\Theta$ is the mean potential temperature, and $T$ is the fluctuation of $\Theta$. The external solenoidal force $\mathbf{f}^{\mathbf{0}}$ mimics the effect of large-scale instabilities and maintains turbulence in a statistically steady state. Note that the temperature Eq. (2) does not involve a separate forcing implying that the temperature fluctuations are excited by the velocity fluctuations.

The spectral domain is bounded by the viscous dissipation wave number $k_{d}=\left(\epsilon / v_{0}^{3}\right)^{1 / 4}$, where $\epsilon$ is the dissipation rate. The Fourier transforms of the velocity and temperature fields are

$$
\begin{aligned}
u_{i}(\mathbf{x}, t) & =\frac{1}{(2 \pi)^{4}} \int_{k \leq k_{d}} \mathrm{~d} \mathbf{k} \int \mathrm{d} \omega u_{i}(\omega, \mathbf{k}) \exp [i(\mathbf{k x}-\omega t)], \\
T(\mathbf{x}, t) & =\frac{1}{(2 \pi)^{4}} \int_{k \leq k_{d}} \mathrm{~d} \mathbf{k} \int \mathrm{d} \omega T(\omega, \mathbf{k}) \exp [i(\mathbf{k x}-\omega t)] .
\end{aligned}
$$

The continuity Eq. (3) takes the form

$u_{\alpha}(\hat{k}) k_{\alpha}=0$,

where $\hat{k} \equiv(\omega, \mathbf{k})$ is a four-dimensional vector in Fourier space. The Fourier-transformed momentum and temperature equations are

$$
\begin{aligned}
\left(-i \omega+v_{0} k^{2}\right) u_{\beta}(\hat{k}) & =f_{\beta}^{0}(\hat{k})-i k_{\beta} \frac{P(\hat{k})}{\rho}+\alpha g T(\hat{k}) \delta_{\beta 3} \\
& -i k_{\mu} \int u_{\beta}(\hat{q}) u_{\mu}(\hat{k}-\hat{q}) \frac{\mathrm{d} \hat{q}}{(2 \pi)^{4}}, \\
\left(-i \omega+\kappa_{0} k^{2}\right) T(\hat{k}) & =-\frac{d \Theta}{d z} u_{3}(\hat{k}) \\
& -i k_{\alpha} \int u_{\alpha}(\hat{q}) T(\hat{k}-\hat{q}) \frac{\mathrm{d} \hat{q}}{(2 \pi)^{4}},
\end{aligned}
$$

where $\hat{q} \equiv(\Omega, \mathbf{q})$. The central difficulty in solving Eqs. (7) and (8) is posed by their nonlinearity represented by the convolution integrals. Another, but less severe problem is caused by their coupling. It would be natural to tackle the nonlinearity by using a perturbative solution based upon the expansion in powers of the Reynolds number Re. However, Re based upon the large-scale flow parameters is very large and the corresponding expansion is strongly divergent.

The approach used in this study is based upon the methodology of the renormalized perturbation theory of turbulence, 
briefly, RPT (McComb, 1991, 1995). The RPT operates with effective, or renormalized, or eddy viscosity and diffusivity rather than their molecular values. In the framework of RPT, the central idea of the present approach can be formulated in terms of the effective Reynolds number: since $R e$ is of the order of $O(1)$ for the smallest scales of motion, one can derive a perturbative solution for these small scales. Then, using this solution, an ensemble averaging is performed over an infinitesimal band of small scale modes. This averaging yields corrections to viscosity and diffusivity giving rise to "effective" or "eddy" viscosity and diffusivity. Then, this procedure is repeated for the next band of the remaining smallest scales, etc. In this process, the small-scale modes are successively purged from the governing equations which thus undergo a gradual coarsening while the effective, based upon the eddy viscosity, Reynolds number $R e$ remains $O(1)$.

2.2 Preconditioning of the momentum and temperature equations

Using the continuity Eq. (6), the pressure term can be excluded in the momentum Eq. (7),

$$
\begin{aligned}
G_{0}^{-1}(\omega, \mathbf{k}) & u_{\beta}(\hat{k})=f_{\beta}^{0}(\hat{k})+\alpha g T(\hat{k}) P_{3 \beta}(\mathbf{k}) \\
& -\frac{i}{2} P_{\beta \mu \nu}(\mathbf{k}) \int u_{\mu}(\hat{q}) u_{\nu}(\hat{k}-\hat{q}) \frac{\mathrm{d} \hat{q}}{(2 \pi)^{4}},
\end{aligned}
$$

where

$$
\begin{aligned}
& P_{i j}(\mathbf{k})=\delta_{i j}-k_{i} k_{j} / k^{2}, \\
& P_{l m n}(\mathbf{k})=k_{m} P_{l n}(\mathbf{k})+k_{n} P_{l m}(\mathbf{k}), \\
& G_{0}^{-1}(\omega, \mathbf{k})=-i \omega+v_{0} k^{2},
\end{aligned}
$$

$G_{0}(\omega, \mathbf{k})$ is the "bare" auxiliary Green function and $\delta_{i j}$ is the Kronecker $\delta$-symbol. The Eq. (9) still contains the temperature on its right side and, thus, is not self-contained. Invoke now the temperature Eq. (8) rewritten as

$$
\begin{aligned}
G_{T 0}^{-1}(\hat{k}) T(\hat{k})= & -\frac{d \Theta}{d z} u_{3}(\hat{k}) \\
& -i k_{\alpha} \int u_{\alpha}(\hat{q}) T(\hat{k}-\hat{q}) \frac{\mathrm{d} \hat{q}}{(2 \pi)^{4}},
\end{aligned}
$$

where $G_{T 0}(\omega, \mathbf{k})$ is the "bare" temperature Green function,

$$
G_{T 0}(\omega, \mathbf{k})=\left(-i \omega+\kappa_{0} k^{2}\right)^{-1} .
$$

Equation (13) represents a forced-dissipative system where the forcing is described by the first term on its right side and the dissipation is included in the Green function. The nonlinear term represents the excitation and damping of the temperature mode $T(\hat{k})$ by all other modes. Let us assume that in the process of small-scale modes elimination, the contribution of the nonlinear term to the forcing is small so that the forcing term preserves its form. The contribution of the nonlinear term to the damping is not small and needs to be calculated. Absorbing this contribution, the diffusivity becomes flow dependent. Due to the anisotropy introduced by stable stratification, the diffusivity is expected to vary differently in the vertical and the horizontal directions. In this representation, the nonlinear Eq. (13) which explicitly describes the interaction between all modes is replaced by a stochastic equation that describes the balance between the excitation and the damping of a single mode and in which the nonlinearity is implicitly included in the damping term,

$T(\hat{k})=-\frac{d \Theta}{d z} G_{T}(\hat{k}) u_{3}(\hat{k})$.

The temperature Green function in Eq. (15) is

$G_{T}(\omega, \mathbf{k})=\left(-i \omega+\kappa_{h} k_{h}^{2}+\kappa_{z} k_{3}^{2}\right)^{-1}$,

where $\kappa_{h}$ and $\kappa_{z}$ are the horizontal and vertical effective diffusivities, and $k^{2}=k_{1}^{2}+k_{2}^{2}+k_{3}^{2}=k_{h}^{2}+k_{3}^{2}$. At the start of the scale elimination process, $\kappa_{h}=\kappa_{z}=\kappa_{0}$, such that $G_{T 0}=G_{T}$. We shall prove the correctness of the formal solution (15) retroactively by showing that this functional form selfreproduces in the process of small-scale modes elimination.

Using Eq. (15), the temperature can be excluded from the momentum Eq. (9) yielding

$$
\begin{aligned}
& {\left[G_{\alpha \beta}^{0}(\hat{k})\right]^{-1} u_{\beta}(\hat{k})=f_{\alpha}^{0}(\hat{k})} \\
& \quad-\frac{i}{2} P_{\alpha \mu \nu}(\mathbf{k}) \int u_{\mu}(\hat{q}) u_{\nu}(\hat{k}-\hat{q}) \frac{\mathrm{d} \hat{q}}{(2 \pi)^{4}},
\end{aligned}
$$

where the "bare" velocity Green function, $G_{\alpha \beta}^{0}(\hat{k})$, has a nondiagonal tensorial structure that reflects the anisotropy introduced by stable stratification,

$$
\begin{aligned}
G_{\alpha \beta}^{0}(\omega, \mathbf{k}) & =G_{0}(\omega, \mathbf{k})\left[\delta_{\alpha \beta}\right. \\
& \left.+N^{2} G_{T}(\omega, \mathbf{k}) G_{0}(\omega, \mathbf{k}) P_{\alpha 3}(\mathbf{k}) \delta_{\beta 3}\right]^{-1} .
\end{aligned}
$$

Similarly to the temperature Eq. (13), Eq. (17) includes the forcing and the nonlinear terms on its right side. The forcing is concentrated on the largest scales of the system and is small otherwise. The effect of the forcing is transmitted to higher and higher wave number modes via nonlinear interactions. The nonlinear interactions are also responsible for the modal damping; every mode loses the same amount of energy it gains due to the forcing. In the process of the smallscale modes elimination, the viscosity undergoes "renormalization." Similarly to the "renormalized," or effective diffusivity, the "renormalized," or effective viscosity is also expected to become anisotropic yielding the effective auxiliary Green function $G(\omega, \mathbf{k})$ in the form

$G(\omega, \mathbf{k})=\left(-i \omega+v_{h} k_{h}^{2}+v_{z} k_{3}^{2}\right)^{-1}$.

Here, $v_{h}=v_{z}=v_{0}$ at the beginning of the scale elimination process. Similarly to the case of the temperature, the correctness of Eq. (19) is proved by demonstrating that this form is self-reproducible in the process of small-scale modes elimination. Since all bare Green functions are equal to their respective effective Green functions at the beginning of the 
scale elimination process, the index ' 0 ' will be removed from all bare Green functions from now on.

The matrix in Eq. (18) can be inverted to yield a general expression for the tensorial velocity Green function $G_{\alpha \beta}(\omega, \mathbf{k})$,

$G_{\alpha \beta}(\omega, \mathbf{k})=G(\omega, \mathbf{k})\left[\delta_{\alpha \beta}+A(\omega, \mathbf{k}) P_{\alpha 3}(\mathbf{k}) \delta_{\beta 3}\right]$,

where the function $A(\omega, \mathbf{k})$ is

$A(\omega, \mathbf{k})=-\frac{N^{2}}{G^{-1}(\omega, \mathbf{k}) G_{T}^{-1}(\omega, \mathbf{k})+N^{2} \sin ^{2} \phi}$,

$N \equiv\left(\alpha g \frac{d \Theta}{d z}\right)^{1 / 2}$ is the buoyancy, or Brunt-Väisälä frequency, and $\phi$ is the angle between $\mathbf{k}$ and the vertical. Note that the term $N^{2} \sin ^{2} \phi$ in the denominator of $A$ generates complex poles. These poles reflect the appearance of internal waves in turbulent flow field.

\subsection{Quasi-Normal Scale Elimination Model (QNSE)}

The formal procedure of the small-scale modes elimination is applied to Eqs. (13) and (17). This procedure requires one to differentiate between the modes designated for purging and the rest of the modes. Assume that all the modes up to a wave number $\Lambda$ have already been eliminated and we now proceed to eliminate the next thin shell $\Delta \Lambda$, where $\Delta \Lambda / \Lambda \ll 1$. Let us define the following domains: $D^{<}=(0, \Lambda-\Delta \Lambda]$, $D^{>}=(\Lambda-\Delta \Lambda, \Lambda]$, and $D=(0, \Lambda]=D^{<} \cup D^{>}$. Then, we define the "slow" $\left(\mathbf{u}^{<}, T^{<}\right)$and "fast" $\left(\mathbf{u}^{>}, T^{>}\right)$velocity and temperature modes in such a way that $k \in D^{<}$for $\mathbf{u}^{<}$and $T^{<}$ while $k \in D^{>}$for $\mathbf{u}^{>}$and $T^{>}$. Finally, $u_{\alpha}$ and $T$ are decomposed into sums, $u_{\alpha}=u_{\alpha}^{<}+u_{\alpha}^{>}$and $T=T^{<}+T^{>}$, which are substituted back in Eqs. (13) and (17) to obtain equations for the slow modes. These equations are not self-contained because they involve terms with fast modes. One can eliminate the fast modes by ensemble-averaging the slow-mode equations over the fast modes in the assumption of quasinormality of the fast modes. The averaging eliminates all the odd moments of the fast modes. The remaining terms contain the velocity correlation, $\left\langle u_{\alpha}(\Omega, \mathbf{q}) u_{\beta}\left(\Omega^{\prime}, \mathbf{q}^{\prime}\right)\right\rangle$. The assumption of statistical homogeneity suggests that the velocity correlation is proportional to $\delta\left(\mathbf{q}+\mathbf{q}^{\prime}\right)$ while the assumption of stationarity yields the proportionality to $\delta\left(\Omega+\Omega^{\prime}\right)$. Thus, the velocity correlation can be written in the form

$$
\begin{aligned}
\left\langle u_{\alpha}(\Omega, \mathbf{q}) u_{\beta}\left(\Omega^{\prime}, \mathbf{q}^{\prime}\right)\right\rangle & =(2 \pi)^{4} \delta\left(\mathbf{q}+\mathbf{q}^{\prime}\right) \delta\left(\Omega+\Omega^{\prime}\right) \\
& \times U_{\alpha \beta}(\Omega, \mathbf{q}),
\end{aligned}
$$

where the coefficient $(2 \pi)^{4}$ is added for convenience. The form of the velocity correlation tensor, $U_{\alpha \beta}(\Omega, \mathbf{q})$, can be established from the general form of the momentum Eq. (17), $u_{\beta}=G_{\beta \alpha} F_{\alpha}$, where $F_{\alpha}$ represents the right side of Eq. (17), the requirement of the incompressibility, and the dimensional considerations,

$U_{\alpha \beta}(\hat{q})=C \bar{\epsilon} q^{-3} G_{\alpha \mu}(\hat{q}) G_{\beta \sigma}^{*}(\hat{q}) P_{\mu \sigma}(\mathbf{q})$,

where $\bar{\epsilon}$ has a meaning of the energy flux through the mode $\mathbf{q}$ and the constant, $C \simeq 26.2$, is found from the balance between the energy gain due to the eddy forcing and the energy loss due to the eddy damping (Sukoriansky et al., 2005b). Strictly speaking, the expression (23) should also be factored with some function of the polar angle $\phi$ but we shall neglect the angular dependence in this study. The ensemble-averaging over the fast modes eliminates a small shell of fast modes, $D^{>}$, from the equations for the slow modes thus shrinking the domain of definition of the slow modes by $\Delta \Lambda$; as a result, the velocity and temperature Green functions receive small gains,

$$
\begin{aligned}
& \Delta G_{\alpha \beta}^{-1}\left(\omega, k, k_{3}\right)=P_{\alpha \mu \theta}(\mathbf{k}) \int^{>} P_{\nu \sigma \beta}(\mathbf{k}-\mathbf{q}) \\
& \times G_{\theta \nu}\left(\omega-\Omega,|\mathbf{k}-\mathbf{q}|, k_{3}-q_{3}\right) U_{\mu \sigma}\left(\Omega, q, q_{3}\right) \frac{\mathrm{d} \mathbf{q} \mathrm{d} \Omega}{(2 \pi)^{4}},
\end{aligned}
$$

$$
\begin{aligned}
& \Delta G_{T}^{-1}\left(\omega, k, k_{3}\right)=k_{\alpha} k_{\beta} \int^{>} U_{\alpha \beta}\left(\Omega, q, q_{3}\right) \\
& \times G_{T}\left(\omega-\Omega, q, q_{3}\right) \frac{\mathrm{d} \mathbf{q} \mathrm{d} \Omega}{(2 \pi)^{4}} .
\end{aligned}
$$

The symbol $\int^{>}$in Eqs. (24), (25) denotes the integration over the shell $D^{>}$. Using Eq. (23), one can evaluate the integrals in Eqs. (24) and (25) and compute these gains; they, in turn, yield corrections to the effective viscosities and diffusivities. The computation is performed in the assumption $k / \Lambda \ll 1$; the details of the integration can be found in Sukoriansky et al. (2005b). Due to anisotropy, these corrections are different in the vertical and the horizontal directions and are proportional to $k_{3}^{2}$ and $k_{h}^{2}$, respectively. The elimination of the shell $D^{>}$from the equations for the slow modes leaves the form of these equations intact such that the same procedure can be repeated to eliminate the next $\Delta \Lambda$ shell. Taking a limit $\Delta \Lambda \rightarrow 0$, a coupled system of four ordinary differential equations for $v_{h}, v_{z}, \kappa_{h}$ and $\kappa_{z}$ is obtained to calculate all corrections. This system can be solved analytically for weak and numerically for arbitrary stratification to obtain scale-dependent, horizontal and vertical eddy viscosities and eddy diffusivities. The integration can be extended to an arbitrary wave number $\Lambda$. If $\Lambda^{-1}$ is smaller than the turbulence macroscale, $k_{L}^{-1}$, then the effective viscosities and diffusivities can be used as subgridscale parameters in largeeddy simulations (LES) where the grid resolution is $\Lambda^{-1}$. Extending $\Lambda^{-1}$ to the integral macroscale, one eliminates all turbulent modes from the equations of motion and arrives at the Reynolds-averaged Navier-Stokes (RANS) description of the system.

Summarizing, let us recap that the present method is based upon the assumption of the quasi-normality of the velocity and temperature fluctuations and the process of successive small-scale modes elimination. To reflect these main features, the method has been coined a quasi-normal scale elimination model, or, briefly, the QNSE model (Sukoriansky et al., 2005b). 


\section{Some results from the QNSE model}

Figure 1 shows the numerical solution to the coupled system of equations for $v_{h}, v_{z}, \kappa_{h}$ and $\kappa_{z}$ given in Sukoriansky et al. (2005b). All eddy viscosities and eddy diffusivities are normalized with $v_{n}$, the eddy viscosity for a neutral flow with the same rate of the viscous dissipation $\epsilon$. The horizontal axis is in the units of the wave number $k$ normalized with the Ozmidov wave number $k_{O}=\left(N^{3} / \epsilon\right)^{1 / 2}$. This figure demonstrates different behaviors of horizontal and vertical eddy viscosities and eddy diffusivities with increasing stable stratification. While the vertical viscosity and diffusivity are suppressed compared to their values in the neutral case, their horizontal counterparts are enhanced. Also, while under the action of strong stable stratification the vertical eddy diffusivity becomes small, the vertical eddy viscosity retains a finite value compared to its corresponding neutral value.

3.1 Modification of the internal wave dispersion relation by turbulence

By solving the equation

$\operatorname{det}\left[G_{\alpha \beta}^{-1}(\omega, \mathbf{k})\right]=0$,

one finds the eigenfrequencies of the system (17); they are given by the real parts of the roots of Eq. (26),

$$
\begin{aligned}
& \omega=\omega_{0}\left\{1-\left(\frac{k}{k_{O}}\right)^{4 / 3}\right. \\
& \left.\times\left[\frac{\left(\frac{\kappa_{z}}{v_{n}}-\frac{v_{z}}{v_{n}}\right) \cos ^{2} \phi+\left(\frac{\kappa_{h}}{v_{n}}-\frac{v_{h}}{v_{n}}\right) \sin ^{2} \phi}{4 \sin \phi}\right]^{2}\right\}^{1 / 2} .
\end{aligned}
$$

Equation (27) provides the dispersion relation for internal waves in the presence of turbulence. In the limit of strong stratification, where $k \rightarrow 0$ or $N \rightarrow \infty$, the classical dispersion relation for linear internal waves, $\omega=N \sin \phi$, is recovered. As the scales decrease, turbulence becomes the dominant dynamical factor; when the wave number $k_{t}(\phi)$, given by

$$
\begin{aligned}
& k_{t}(\phi)=k_{O}\left|\frac{4 \sin \phi}{\left(\frac{\kappa_{z}}{v_{n}}-\frac{v_{z}}{v_{n}}\right) \cos ^{2} \phi+\left(\frac{\kappa_{h}}{v_{n}}-\frac{v_{h}}{v_{n}}\right) \sin ^{2} \phi}\right|^{3 / 2} \\
& \simeq 32 k_{O}|\sin \phi|^{3 / 2},
\end{aligned}
$$

is reached, the turbulent overturn completely overwhelms the generation of waves, such that $k_{t}(\phi)$ provides a threshold scale at which the internal waves disappear. The waves can only exist inside the torus $k \leq k_{t}(\phi)$. One can see that $k_{t}(\phi)$ reaches its maximum at $\phi= \pm \pi / 2$. This value is shown in Fig. 1 by a vertical straight line. It is easy to see that the threshold of the internal wave generation is in the range where the eddy viscosities and eddy diffusivities just begin to deviate from their values under neutral stratification, i.e., the threshold (28) coincides with the beginning of the flow anisotropization under the action of stable stratification.

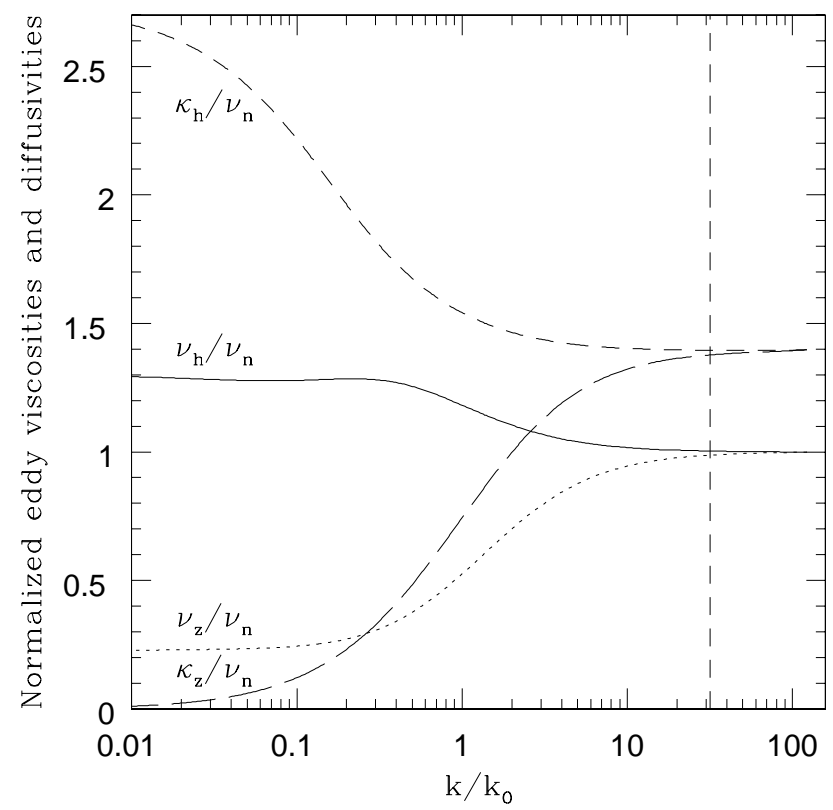

Fig. 1. Horizontal and vertical eddy viscosities and diffusivities normalized with the corresponding value of the eddy viscosity in the neutral case, $v_{n}$, as functions of $k / k_{O}, k_{O}=\left(N^{3} / \epsilon\right)^{1 / 2}$ is the Ozmidov wave number. The dashed vertical line indicates the maximum wave number threshold of internal wave generation in the presence of turbulence, Eq. (28).

\subsection{The asymptotic case of weak stable stratification}

For weakly stable stratification, $\left(k / k_{O}\right)^{-1} \ll 1$, and one can use this ratio as a small parameter in power series expansions of the eddy viscosities and eddy diffusivities. Retaining only the first term in these expansions, we obtain

$$
\begin{aligned}
v_{h} / v_{n} & =1+0.38\left(k / k_{O}\right)^{-4 / 3}, \\
v_{z} / v_{n} & =1-1.24\left(k / k_{O}\right)^{-4 / 3}, \\
\kappa_{h} / v_{n} & =\alpha+0.22\left(k / k_{O}\right)^{-4 / 3}, \\
\kappa_{z} / v_{n} & =\alpha-1.6\left(k / k_{O}\right)^{-4 / 3},
\end{aligned}
$$

where $\alpha^{-1}=P r_{t 0} \simeq 0.72$ is the turbulent Prandtl number for neutral flows (Sukoriansky et al., 2005b). These equations show that the normalized horizontal eddy viscosity and diffusivity tend to increase with increasing stratification while their vertical counterparts decrease thus pointing to the largescale anisotropization of the turbulent transport of momentum and heat in stably stratified flows. Equations (29)-(32) can be used to quantify the onset of this anisotropization; for instance, at $k / k_{O} \simeq 6$, all effective viscosities and diffusivities differ from their neutral values by less than $10 \%$.

\subsection{Turbulence spectra}

Due to the flow anisotropy, a traditional 3-D energy spectrum provides only limited information; as shown in Sukoriansky et al. (2005b), it remains close to the classical Kolmogorov spectrum. Various 1-D spectra provide more detailed information on flow anisotropization under the action of stable 

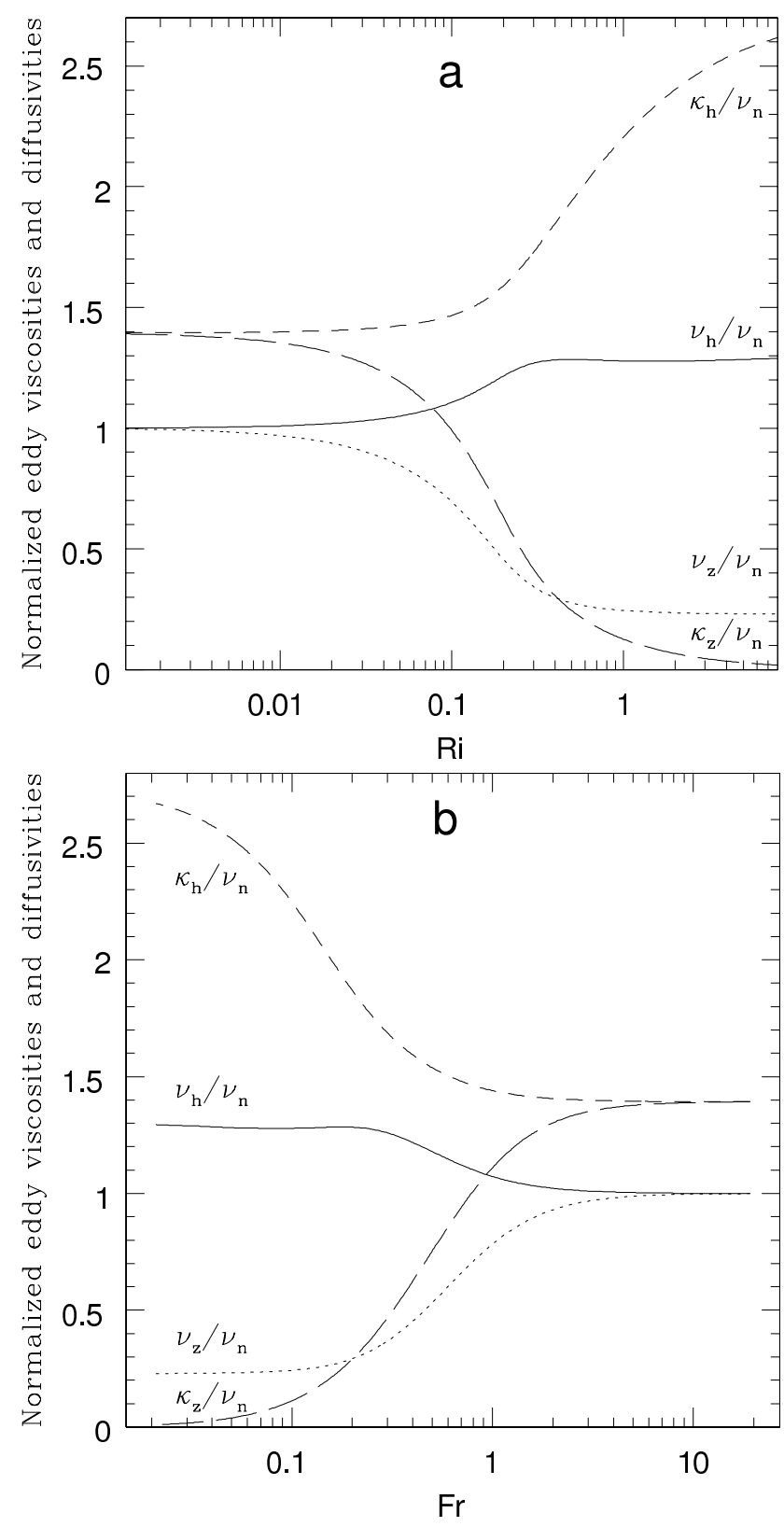

Fig. 2. Horizontal and vertical turbulent exchange coefficients normalized with $v_{n}$ as functions of $\operatorname{Ri}(\mathbf{a})$ and $\operatorname{Fr}(\mathbf{b})$.

stratification. In the limit of weak stratification, these spectra can be computed analytically using Eqs. (23) and (29)-(32). The vertical spectrum of the horizontal velocity then takes the form

$$
\begin{aligned}
E_{1}\left(k_{3}\right) & =\frac{8}{(2 \pi)^{4}} \int U_{11}(\omega, \mathbf{k}) \mathrm{d} \omega \mathrm{d} k_{1} \mathrm{~d} k_{2} \\
& =0.626 \epsilon^{2 / 3} k_{3}^{-5 / 3}+0.214 N^{2} k_{3}^{-3} .
\end{aligned}
$$

Equation (33) shows that the QNSE model reproduces the $N^{2} k_{3}^{-3}$ spectrum; the transition from the Kolmogorov $-5 / 3$ to the stable stratification dominated -3 spectrum takes place on scales of the order of the Ozmidov scale. The coefficients in Eq. (33) are in very good agreement with experimental data (Gargett et al., 1981) and LES (Carnevale et al., 2001) where the factor with the term $N^{2} k_{3}^{-3}$ was found to be about 0.2 .

For other 1-D spectra one obtains:

$$
\begin{aligned}
E_{3}\left(k_{1}\right) & =\frac{8}{(2 \pi)^{4}} \int U_{33}(\omega, \mathbf{k}) \mathrm{d} \omega \mathrm{d} k_{2} \mathrm{~d} k_{3} \\
& =0.626 \epsilon^{2 / 3} k_{1}^{-5 / 3}-0.704 N^{2} k_{1}^{-3}, \\
E_{3}\left(k_{3}\right) & =\frac{8}{(2 \pi)^{4}} \int U_{33}(\omega, \mathbf{k}) \mathrm{d} \omega \mathrm{d} k_{1} \mathrm{~d} k_{2} \\
& =0.47 \epsilon^{2 / 3} k_{3}^{-5 / 3}-0.143 N^{2} k_{3}^{-3} .
\end{aligned}
$$

The anisotropization of the flow field manifests itself as energy increase in the horizontal velocity components at the expense of their vertical counterpart.

\section{RANS modeling}

In the conventional RANS modeling, equations of motion are Reynolds-averaged and then a system of closure assumptions is introduced to derive expressions for eddy viscosity and eddy diffusivity, $K_{M}$ and $K_{H}$ (see, e.g., Mellor and Yamada, 1982). The sum of the diagonal Reynolds stresses is used to obtain the equation for the total kinetic energy, $K$, and then one more equation is required to specify the turbulence macroscale, $L$, or, equivalently, the rate of the turbulent dissipation, $\epsilon$. The latter equation cannot be derived analytically and is, thus, postulated. The distribution of $L$ can be prescribed by either an algebraic equation leading to the family of one-equation models, or by a differential equation usually constructed following the template of the energy equation and leading to the family of two-equation models. Thus, the RANS models involve two components that require ad hoc approximations - expressions for eddy viscosities and eddy diffusivities and an equation for $L$ or $\epsilon$.

The use of the QNSE methodology to derive the RANStype models yields expressions for the eddy viscosities and eddy diffusivities directly from the theory and thus allows one to bypass the use of the Reynolds stress closure assumptions. An equation for $L$ or $\epsilon$, on the other hand, still needs to be postulated. It is hypothesized, however, that the QNSEbased expressions for $v_{h}, v_{z}, \kappa_{h}$ and $\kappa_{z}\left(v_{z}\right.$ and $\kappa_{z}$ are identified with $K_{M}$ and $K_{H}$, respectively) can significantly improve the performance of the RANS models. In this and the following sections, we shall substantiate this hypothesis by testing the QNSE-based RANS models against various experimental and field data.

When the process of small-scale modes elimination in the QNSE model is extended to the wave number corresponding to the turbulence macroscale, $k_{L}^{-1}$, the RANS-type equations are obtained. The details of derivation of the RANS models based upon the QNSE methodology are given in Sukoriansky et al. (2005b). Very briefly, this derivation is based upon the theoretically obtained expression for the total kinetic energy spectrum. Integrating this expression to $k_{L}$, a key relationship between the total kinetic energy, 


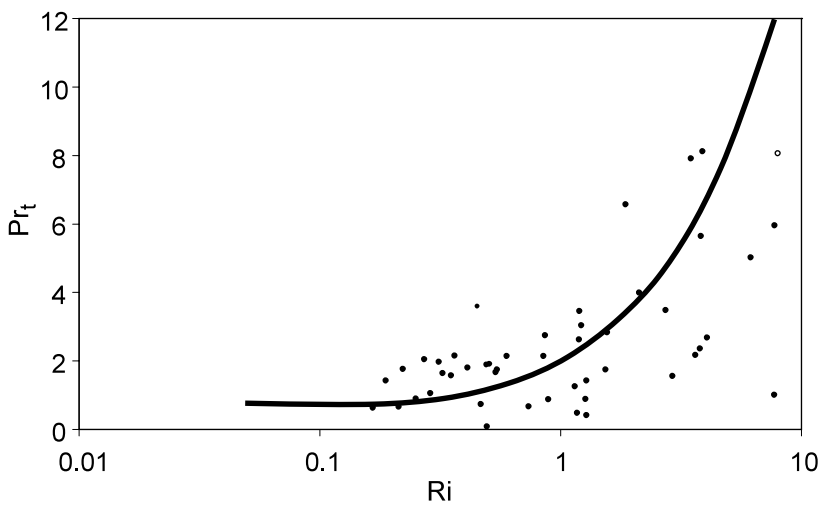

Fig. 3. Vertical turbulent Prandtl number as a function of $R i$. Data points are laboratory measurements from Huq and Stewart (private communication); the solid line represents QNSE model's results.

$K$, the rate of the energy transfer, $\bar{\epsilon}$, and $k_{L}$ is derived. These parameters can be utilized to form the Froude number, $F r=\epsilon / N K$, which characterizes the strength of stratification. Note that $\mathrm{Fr}$ can be used in both shear and shearfree flows. If the source of turbulence is the velocity shear with the mean strength $S$, then the gradient Richardson number, $R i=N^{2} / S^{2}$, can be introduced as an alternative measure of the strength of stratification. Determining the value of $\bar{\epsilon}$ as a difference between the energy production due to the shear, $P=v_{z} S^{2}$, and the buoyancy destruction, $B=\kappa_{z} N^{2}$, find $\bar{\epsilon}=P-B=v_{z} S^{2}-\kappa_{z} N^{2}=S^{2}\left(v_{z}-\kappa_{z} R i\right)$. This expression enables one to relate $R i$ to $F r$ such that the vertical eddy viscosity and eddy diffusivity can be expressed as functions of either $R i$ or $F r$, Fig. 2.

The inspection of Fig. 2 reveals that

(i) for $R i>0.1$, both vertical viscosity and diffusivity decrease, with the diffusivity decreasing faster than the viscosity (supposedly, due to the mixing from internal gravity waves);

(ii) while the vertical eddy diffusivity becomes small with increasing stratification, the vertical eddy viscosity remains equal to about $25 \%$ of its neutral value. This behavior indicates that internal waves are more effective in mixing the momentum than the scalar;

(iii) the horizontal mixing increases with $R i$; the model accounts for the increasing flow anisotropization;

(iv) the crossover from neutral to stratified flow regime is replicated as a sharp drop in the vertical eddy viscosities and eddy diffusivities compared to their neutral values.

One of the general conclusions that may be drawn from these results is that a single valued critical Richardson number at which turbulence is abruptly suppressed does not exist. Instead, there exists a gradual turbulence - internal wave transition. This result is consistent with the abundant volume of data collected in meteorological and oceanographic observations (see, e.g., Kondo et al., 1978; Yagüe and Cano, 1994; Yagüe et al., 2001; Strang and Fernando, 2001; Monti et al., 2002; Mahalov et al., 2004; Mack and Schoeberlein, 2004, and many others) in which turbulence survived in flows with

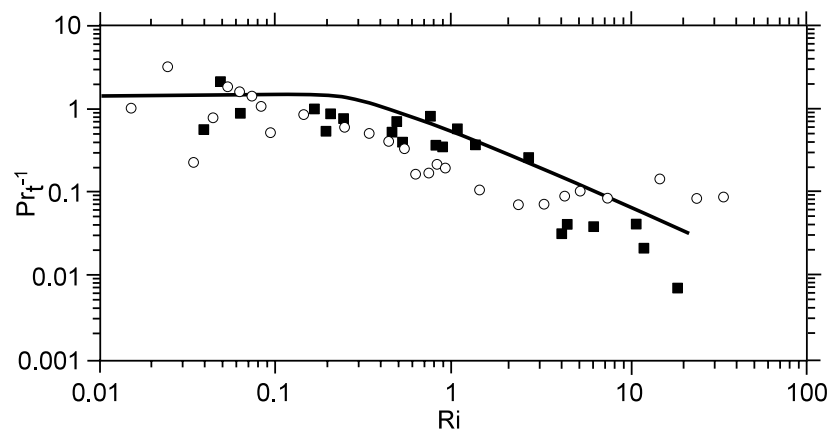

Fig. 4. Inverse Prandtl number, $P r_{t}^{-1}=\kappa_{z} / v_{z}$, as a function of $R i$. Experimental data points are from Monti et al. (2002).

$R i$ far exceeding the critical value of $1 / 4$ obtained from the linear stability analysis by Miles (1961) and Howard (1961) or 1 that follows from the nonlinear analysis by Abarbanel et al. (1984).

\section{Comparison with experimental and observational data and numerical simulations}

5.1 The vertical turbulent Prandtl number and the flux Richardson number

The vertical turbulent Prandtl number is an important characteristic of momentum and temperature mixing under the action of stable stratification. The significance of the accurate representation of $\mathrm{Pr}_{t}$ has been underscored in recent numerical simulations by Noh et al. (2005) who have shown that the ocean general circulation models provide more realistic description of the equatorial mixed layer, deep Equatorial Undercurrent, and the zonal slope of the thermocline when they incorporate growing with stratification vertical Prandtl number.

We have compared QNSE model predictions for $P r_{t}$ as a function of the gradient Richardson number, $R i$, with laboratory data by Huq and Stewart (private communication), in Fig. 3 and observations in stably stratified atmospheric boundary layers by Monti et al. (2002) in Fig. 4. In both cases the agreement was very good for all available Richardson numbers up to $R i \sim 10$. As was explained in Sect. 4, the derivation of the RANS equations is based upon the theoretical expression for the total kinetic energy integrated to $k_{L}$. In real flows, the spectral behavior on large scales in the vicinity of $k_{L}^{-1}$ is not universal such that the above relationship between $K, \epsilon$ and $k_{L}$ may need to be adjusted by introducing an experimentally determined factor. This adjustment would be equivalent to calibration of the values of $F r$ or $R i$. We have used a factor of 0.5 with $R i$ to compare our results for $P r_{t}^{-1}$ with the data by Yaguie et al. (2001) collected at Halley Base, Antarctica. The comparison in Fig. 5 shows good agreement between the model and the data. The same factor would have improved the agreement with the data in Fig. 4 


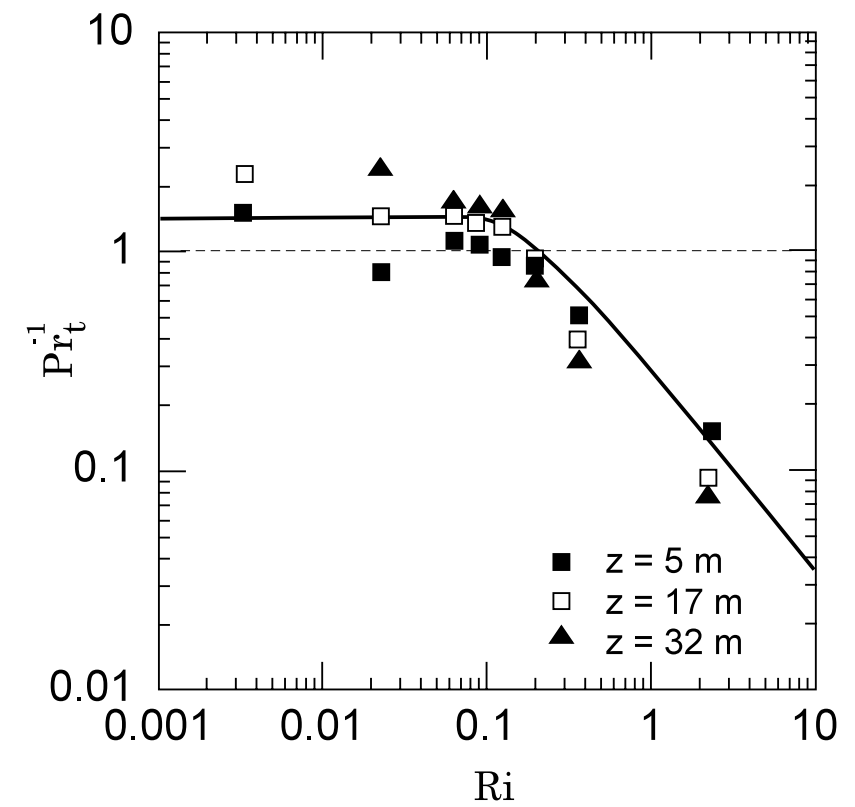

Fig. 5. Inverse Prandtl number, $P r_{t}^{-1}=\kappa_{z} / v_{z}$, as a function of $R i$. The observational data points are from Halley Base, Antarctica (Yagüe et al., 2001).

as well but a more detailed exploration of this issue requires further research.

Another characteristic of stably stratified turbulent flows is the flux Richardson number, $R i_{f}=B / P=R i / P r_{t}$, which sometimes is referred to as the mixing efficiency. This parameter is often used to characterize the eddy diffusivities. The relationship between $R i_{f}$ and $R i$ has been a subject of an extensive research. Figures 4 and 5 indicate that at large $R i$, the present model yields $P r_{t} \propto R i$ such that $R i_{f}$ reaches a maximum of about 0.5 at $R i \simeq 1.5$ when the aforementioned correcting factor of 0.5 with $R i$ is not used and about 0.3 when this correction is employed. This theoretical behavior of $R i_{f}$ agrees with some other theories and data sets. Some data sets, e.g., Monti et al. (2002), show a decrease of $R i_{f}$ for $R i>2$. The latter paper also presents a relatively recent review of data sets and theories regarding $R i_{f}$.

\subsection{Composite spectrum of the vertical shear in the upper ocean}

The composite spectrum of the vertical shear in the upper ocean was compiled in Gargett et al. (1981) but has never been obtained theoretically. Figure 6 compares the observational data with the spectrum (33) derived analytically from the QNSE model in the limit of weak stable stratification, $k / k_{O} \geq 1$. Note that although Eq. (33) has been obtained in the asymptotics $k / k_{O} \geq 1$, we have extended this solution beyond that range for illustration of the -3 slope.

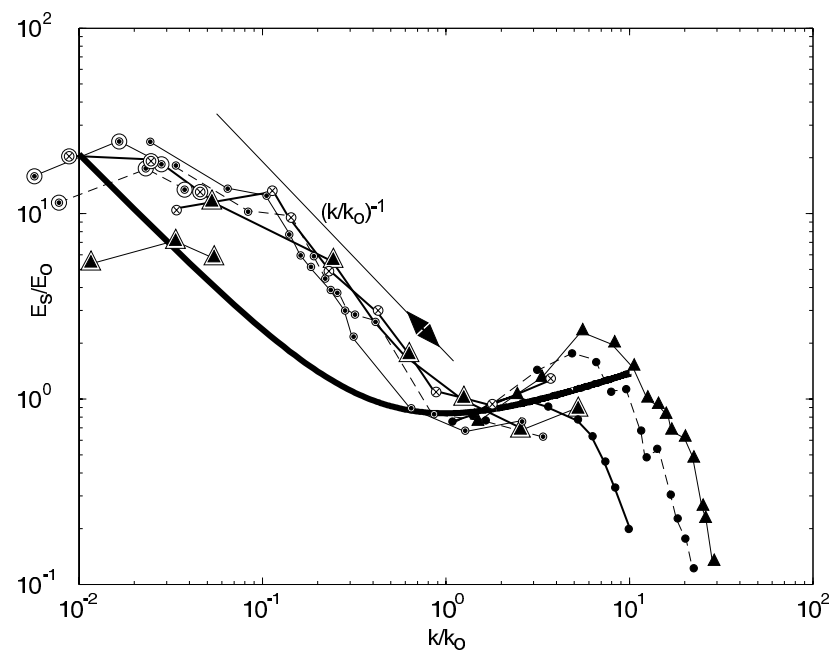

Fig. 6. Composite spectrum of the vertical shear in the upper ocean: the data is from Gargett et al. (1981), the solid line is based upon Eq. (33).

\section{A new $K-\epsilon$ model}

Based upon the QNSE theory, we have developed a new $K-\epsilon$ model (Sukoriansky et al., 2005a). The effect of stratification was incorporated in the $\epsilon$-equation similarly to the effect of rotation in the model by Detering and Etling (1985). The new model has been implemented in the 1-D version of the weather forecast model HIRLAM (high-resolution, limited area model) described in Perov and Gollvik (1996) and Perov et al. (2001) to simulate a stably stratified atmospheric boundary layer $(\mathrm{ABL})$. The resulting model has been tested against observational data collected in several campaigns. Here we show several examples of these comparisons in order to demonstrate good agreement between the data and the QNSE-based $K-\epsilon$ model predictions.

\subsection{Comparison with the data from CASES-99}

The Cooperative Atmosphere-Surface Exchange Study-1999 (CASES-99) campaign took place in southeastern Kansas in the fall of 1999; the data description can be found in Poulos et al. (2002). Here, we are concerned with an event that occurred over the night of 20-21 October 1999, during the intensive observation period (IOP) 9. The starting time was on 22:00 UTC (Greenwich Mean Time) on 20 October and the ending time was on 13:00 UTC $($ UTC $=$ Local time $+5 \mathrm{~h}$ ) on 21 October. The temperature and wind speed profiles at the beginning of the event are the green dashed lines in Fig. 7a and b, respectively. During IOP-9, the surface temperature decreased from $293 \mathrm{~K}$ to $278 \mathrm{~K}$. After $4-5 \mathrm{~h}$ from the beginning, a well-developed low-level jet (LLJ) was formed, shown by blue asterisks in Fig. 7b; it attained a maximum speed of about $14 \mathrm{~m} \mathrm{~s}^{-1}$ at an altitude of approximately $120 \mathrm{~m}$. Within a span of an hour, between 06:30 UTC and 07:30 UTC, the wind speed evolved from a narrow LLJ to a profile that increased with height from the ground to a point somewhat higher than the earlier LLJ peak, 
with roughly constant wind speed $\left(9-12 \mathrm{~m} \mathrm{~s}^{-1}\right)$ above that height, shown by the red crosses in Fig. 7b. Some of the temperature measurements made during that period showed the development of an extremely steep positive temperature gradient near 180-190 m. As recorded in Balsley et al. (2003), the change of temperature could reach $1 \mathrm{~K}$ over only $5 \mathrm{~cm}$ altitude which would correspond to a vertical temperature gradient of $20 \mathrm{~K} \mathrm{~m}^{-1}$. This sharp temperature gradient remained for at least next $20 \mathrm{~min}$. Balsley et al. (2003) note that such a steep temperature gradient exceeds all previously observed gradients in the atmospheric boundary layers (see, for instance, Yagüe and Cano, 1994; Yagüe and Redondo, 1995; Yagüe et al., 2001, for ABLs at Halley Base in Antarctica) by over an order of magnitude.

We have simulated the IOP-9 event with the new $K-\epsilon$ model in a single-column format, where measured vertical profiles of wind velocities and potential temperature at 23:00 UTC were used as initial conditions. Figure 7 compares the simulated temperature and velocity profiles with those measured during IOP-9. One can see that the agreement between the model and the data is very good, in particular that the model reproduces LLJ, although the latter could only be replicated by introducing a horizontal advection of turbulent kinetic energy. Since we are operating with a one-dimensional model, processes responsible for the horizontal advection cannot develop in the model and can only be introduced as external parameters. We have included the horizontal advection of turbulence kinetic energy in our onecolumn simulations based upon the following citation from the "IOP Summary Report" for IOP-9: "At one point, an apparent 'fossil turbulence' event some $700 \mathrm{~m}$ deep and elevated at $\sim 500 \mathrm{~m}$ above ground level seemed to advect into the area. The King Air flew at 3 levels through this event and the NOAA HRDL, in vertical stare, captured vertical velocities of $50-60 \mathrm{~cm} \mathrm{~s}^{-1}$. This was not a wave-like or developed strong shear both above and below the jet. Sometime after midnight (local time) the jet nearly disappeared over the site, apparently it mixed out. The jet was re-established by 03:00 (local time, 08:00 UTC), October 21."

Another surprising feature during IOP-9 was the steepness of the gradients of turbulence characteristics at the top of the nocturnal boundary layer (NBL) and inside the residual layer (RL) defined as a region above the NBL that usually bears slight stable stratification and extends upward reaching the height affected by the signature of the capping inversion from the previous day. At the NBL top $(z=200 \mathrm{~m}$ in Fig. 8, the rate of the energy dissipation and temperature structure parameters dropped sharply exceeding one order of magnitude over an altitude of only a few meters. At higher altitudes within the RL, a region of very weak turbulence was observed whose thickness was about $60 \mathrm{~m}$. This low-turbulence region exhibited sharp edges at which $\epsilon$ and temperature structure parameters could change by an order of magnitude and more over vertical distances of only a few meters. Figure 8 demonstrates that our new $K-\epsilon$ model can reproduce an irregular behavior of $\epsilon$ with a good degree of accuracy.
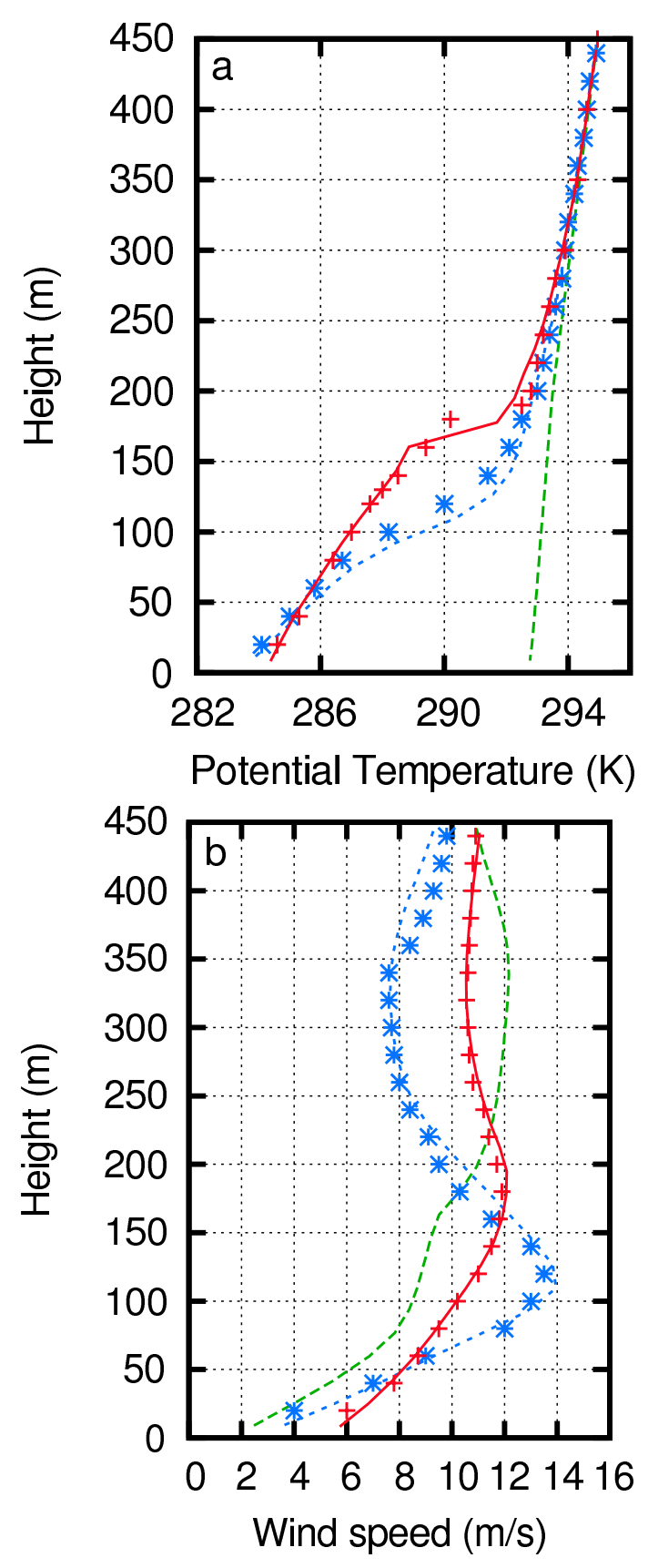

Fig. 7. Evolution of the potential temperature (a) and wind (b) profiles in CASES-99 as simulated by the new $K-\epsilon$ model. The green dashed lines - initial profiles at 23:00 UTC; the blue asterisks - observations at 06:30 UTC; the blue dotted lines - model predictions at 0630 UTC; the red crosses - observations at 07:30 UTC; the red solid lines - model predictions at 07:30 UTC.

Summarizing the simulations of the IOP-9 of CASES-99, we note that the new $K-\epsilon$ model is capable of faithfully replicating complicated processes that take place in NBLs including fast formation and disappearance of the low-level jets, sharp temperature gradients, and irregular and sharply changing vertical profiles of turbulence characteristics. 


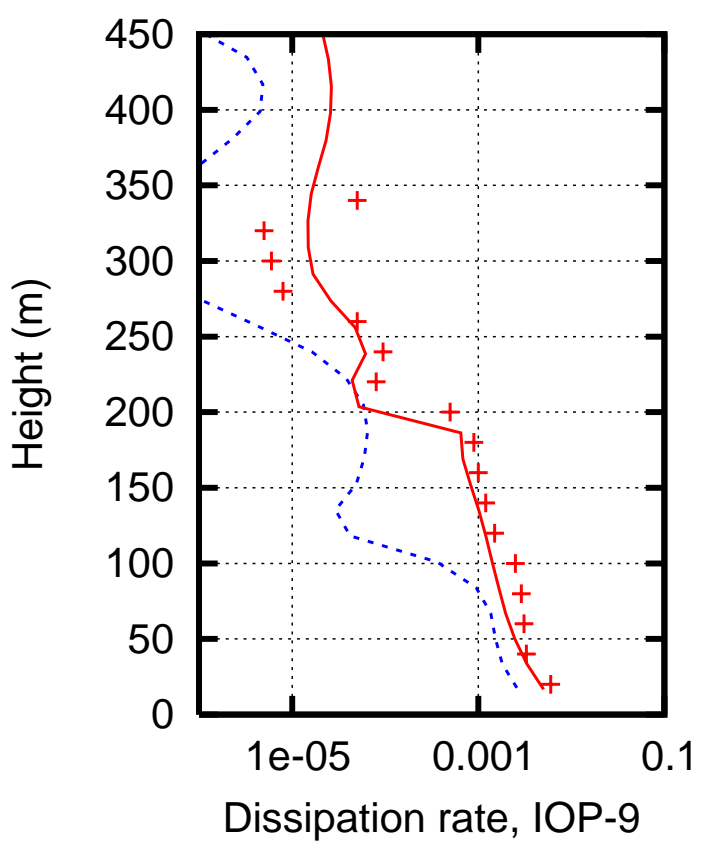

Fig. 8. Vertical profiles of the dissipation rate $\epsilon$ during IOP-9 of CASES-99. Red crosses - observations at 07:30 UTC; blue dotted line and red solid line are model predictions at 06:30 and 07:30 UTC, respectively.

\subsection{Comparison with the data from BASE}

The goal of BASE (Beaufort Arctic Storms Experiment) was to improve understanding of the Arctic weather systems during the fall season. Accordingly, BASE was conducted from 19 September through 29 October 1994 in the Beaufort Sea. The data description can be found in Curry et al. (1996). The data from BASE was successfully simulated in LES by Kosovic and Curry (2000) who considered two cases which differed by the rate of the surface cooling equal to $0.25 \mathrm{~K} \mathrm{hr}^{-1}$ (a moderately stable ABL, Fig. 9a) and $1.0 \mathrm{~K} \mathrm{hr}^{-1}$ (a strongly stable ABL, Fig. 9b). The strength of the overlying inversion was $0.01 \mathrm{~K} \mathrm{~m}^{-1}$ and the surface roughness was $0.1 \mathrm{~m}$. The LES simulated the transitional process of the boundary layer adjustment to the respective surface cooling rates for each of the two cases.

Using the new QNSE-based $K-\epsilon$ model, we have simulated these two experiments in a single-column formulation with a vertical resolution of $10 \mathrm{~m}$. Both simulations were executed for $12 \mathrm{~h}$ of physical time during which the flow attained a quasi-steady state. The initial values of the turbulent kinetic energy and dissipation were set equal to small constants. In the course of the simulations, the model "forgot" the initial distributions of the turbulence energy $K$ and $\epsilon$ after approximately one hour of integration.

Figure 9 shows the profiles of the potential temperature (PT) simulated with the new and the standard $K-\epsilon$ models as well as with the LES after $12 \mathrm{~h}$ simulation. The agreement of the new model with the LES data is good for the case of moderate stratification. The standard model strongly
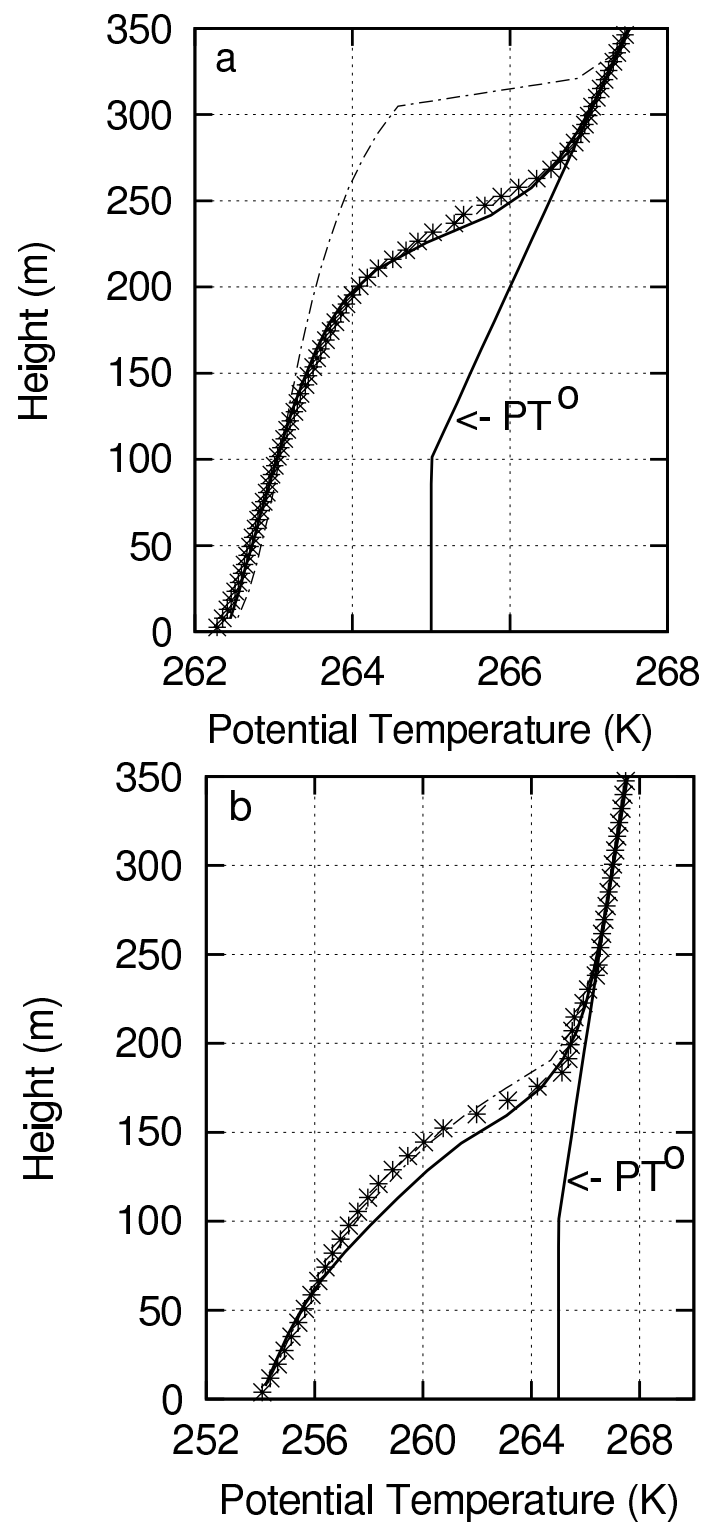

Fig. 9. Vertical profiles of mean potential temperature (PT) for the cases of moderate (a) and strong (b) stable stratification simulated with the new (solid line) and standard (dashed-dotted line) $K-\epsilon$ models. The LES results by Kosovic and Curry (2000) are shown by asterisks. The initial PT profiles (marked as $\mathrm{PT}^{0}$ ) are shown by straight solid lines.

overestimates the height of the temperature boundary layer in that case. In the case of strong stratification, the simulated temperature profile is lower than in LES, which ignored the effect of stable stratification on the sub-grid scale (SGS) viscosities and diffusivities by adopting the parameters from the neutral case. As can be seen on Fig. 1, such an approximation is justified when the grid size is smaller than the Ozmidov length scale, $L_{O}=\pi / k_{O}$. However, in strong stratification, $L_{O}$ decreases and may become comparable to or even smaller than the grid size. The eddy viscosities and diffusivities in this case are significantly reduced compared 

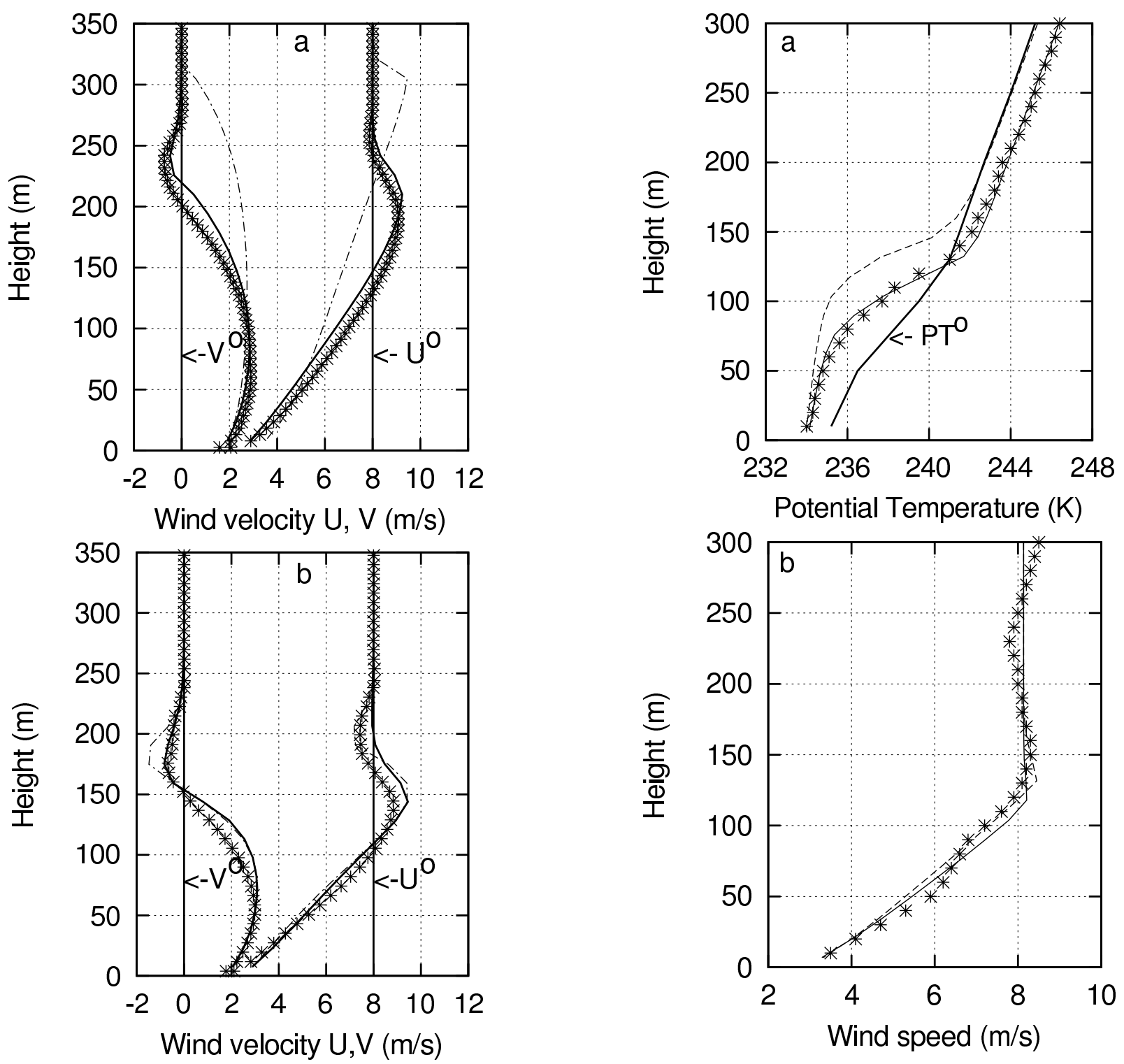

Fig. 10. Vertical profiles of mean horizontal wind components, $U$ and $V$, simulated with the new and standard $K-\epsilon$ models. The initial profiles are marked as $U^{0}$ and $V^{0}$, respectively. The order of the panels and the description of the lines and asterisks are the same as in Fig. 9.

to their neutral values. The use of these values in strongly stratified flows may result in overestimated mixing and overpredicted boundary layer height. Since the eddy diffusivity in such flows decreases much faster than the eddy viscosity, the overprediction of the temperature boundary layer height can be expected to be most profound. To avoid this problem, one can either increase the grid resolution or account for the effect of stratification on SGS parameters (Sukoriansky et al., 2005a).

The vertical profiles of the horizontal wind components, $U$ and $V$, are shown in Fig. 10. The results obtained with the new $K-\epsilon$ model show good agreement with those produced in LES by Kosovic and Curry (2000). The standard $K-\epsilon$

Fig. 11. Vertical profiles of potential temperature (a) and wind speed (b) in SHEBA experiment. The observational data is represented by asterisks, the simulations with the new $K-\epsilon$ model are shown by thin solid lines. The short dashed lines refer to the case in which the vertical advection is not accounted for.

model does not perform well in the case of the moderate stratification but under strong stratification its performance improves significantly.

\subsection{Comparison with the data from SHEBA}

During the Surface Heat Budget of the Arctic Ocean (SHEBA) experiment, an array of instrument sites was arranged around an icebreaker frozen in the Arctic Ocean pack ice (Uttal et al., 2002; Andreas et al., 1999). Over the year 1998, the entire field camp drifted together with the pack ice across the Beaufort and Chukchi seas for approximately $1500 \mathrm{~km}$. A large volume of atmospheric and surface data 


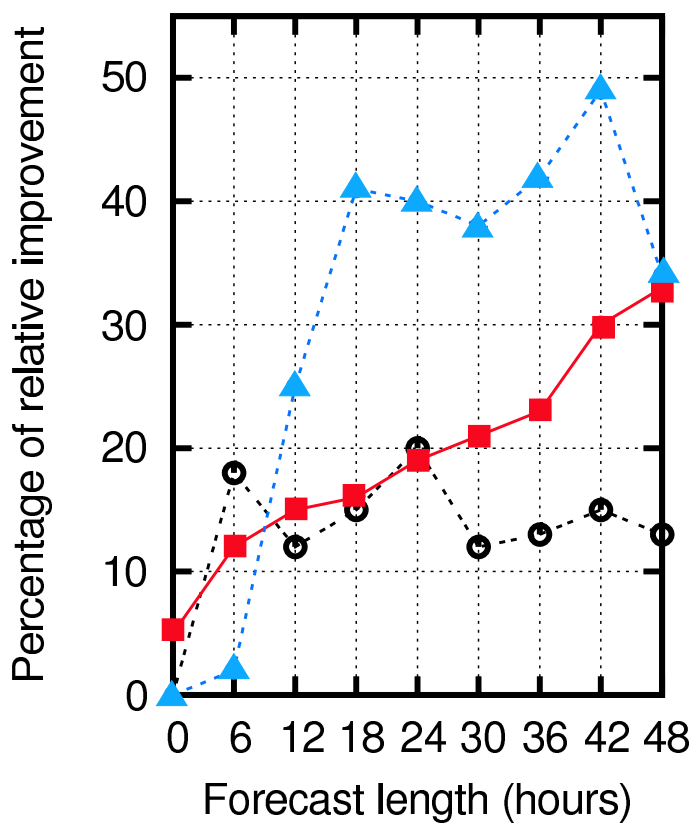

Fig. 12. Percentage of relative improvement in $+48 \mathrm{~h}$ weather forecast over Scandinavia using QNSE-based eddy viscosity and eddy diffusivity in HIRLAM. Black dotes and red squares and corresponding lines refer to temperature and relative humidity at $2 \mathrm{~m}$ height, respectively, and blue triangles and line denote the mean sea-level pressure.

was recorded. One of the main objectives of the SHEBA experiment was to collect surface and ABL data suitable for evaluation of the parameterization schemes employed in global circulation and numerical weather prediction models for high latitudes. The present model-data comparisons, shown in Fig. 11, were performed for the winter case of 15 January, from 0 to $12 \mathrm{~h}$. The initial time was characterized by strong stability, where the low surface temperature was $T_{s}=235 \mathrm{~K}$ and the geostrophic wind velocity was $U_{g}=6 \mathrm{~m}$ $\mathrm{s}^{-1}$. During the span of $12 \mathrm{~h}, U_{g}$ increased to $8.2 \mathrm{~m} \mathrm{~s}^{-1}$ and $T_{s}$ decreased to $233.5 \mathrm{~K}$. This process was simulated using the new $K-\epsilon$ model in a single-column format; the results of the $12 \mathrm{~h}$ integration are shown in Fig. 11. The observed vertical profile of potential temperature above ABL had shifted during the integration period relative to the initial profile. The analysis of the ECMWF weather forecast for this period has shown a week subsidence (negative vertical velocity) over the area of study. To reflect the ensuing processes of the large-scale vertical advection of potential temperature and velocity, a negative vertical velocity of $-10^{-3} \mathrm{~m} \mathrm{~s}^{-1}$ was incorporated in the model. With vertical advection accounted for, the simulated potential temperature and velocity profiles are in good agreement with the observational data. Figure 11 demonstrates that the data cannot be reliably reproduced if the vertical advection is not accounted for.

\section{Testing of $v_{z}$ and $\kappa_{z}$ in a high-resolution weather pre- diction model}

We have tested the QNSE-model derived vertical eddy viscosity and eddy diffusivity in the numerical weather prediction system based upon HIRLAM which is being routinely used for operational weather prediction in Europe. The model's grid covers the North-East Atlantic, Europe, and Greenland. HIRLAM is a hydrostatic model with $438 \times 336$ points in the horizontal that provide $22 \mathrm{~km} \times 22 \mathrm{~km}$ resolution, and 40 levels in the vertical. Lateral boundary conditions and the first guess field are adopted from the European Centre (ECMWF) operations. HIRLAM employs massive data assimilation that utilizes data from over 1000 stations all over Europe; the data assimilation cycle is $6 \mathrm{~h}$. Weather forecasts are obtained by executing the HIRLAM code for $48 \mathrm{~h}$ from the initial times at 00:00, 06:00, 12:00, 18:00 UTC. In total, for a one-month diagnostics, some $120,+48 \mathrm{~h}$ forecasts are available. These data sets are used for statistical analysis and calculation the skills of the forecast. We have chosen to run such diagnostics for the month of January, 2005 because it was a relatively cold month with significant signature of strong stable stratification.

To test the QNSE-based vertical eddy viscosity and eddy diffusivity, $v_{z}$ and $\kappa_{z}$, shown on Fig. 1, against their conventional counterparts employed in HIRLAM, we have compared one-month statistical skills of parallel simulations that used both formulations. The difference between the simulations was only in the formulation of the vertical eddy viscosity and eddy diffusivity for the case of stable stratification; the rest of the model was left intact. Although the model in these simulations was run over the entire computational domain, we have concentrated on its predictions over Scandinavia only as this area was strongly affected by the cold weather. Figure 12 summarizes the results of the parallel experiment; it shows the percentage of relative improvement (PRI) in $+48 \mathrm{~h}$ weather forecast for the bias of the sea-level pressure as well as those of the temperature and relative humidity at the height of $2 \mathrm{~m}$. The PRI was calculated as (new bias - reference bias)/(reference bias). As one can see, the new stability functions significantly improve the predictive skills of HIRLAM for stably stratified atmospheric boundary layers.

\section{Conclusions}

The basic assumption of the new spectral model of turbulence presented in this paper, the QNSE model, is the hypothesis of quasi-normality which leads to a self-contained procedure of consecutive coarse-graining. The model explicitly resolves the stable stratification-induced disparity between the transport processes in the horizontal and vertical directions and accounts for the combined effect of turbulence and waves. Anisotropic turbulent viscosities and diffusivities obtained from the model are in good agreement with experimental data. Various anisotropic spectra can be calculated 
analytically in the limit of weak stratification; one of the results is the demonstration that the energy of the horizontal flow components increases at the expense of their vertical counterpart. For the first time, the transition from the Kolmogorov to the $N^{2} k_{3}^{-3}$ spectral laws for the one-dimensional spectrum of the horizontal velocity, $E_{1}\left(k_{3}\right)$, has been derived analytically. The QNSE model yields modification of the classical dispersion relation for internal waves that accounts for turbulence. The QNSE theory provides subgridscale parameterizations that can be used in both LES and RANS models. The QNSE approach has been implemented in a $K-\epsilon$ model of a stratified ABL; good agreement with CASES99, BASE and SHEBA datasets has been demonstrated for cases of moderate and strong stable stratification. When implemented in the numerical weather forecast system HIRLAM, the QNSE-based vertical eddy viscosity and eddy diffusivity substantially improve the system's predictive skills. The QNSE model indicates that there is no single Richardson number at which turbulence is completely suppressed. Instead, there exists a range of $R i$ where the vertical eddy viscosity and eddy diffusivity undergo dramatic decrease but turbulence survives even at very large values of $R i$. The QNSE-based $K-\epsilon$ model can be applied to engineering flows and $\mathrm{ABL}$ with and without effects of rotation and stratification using invariant set of constants. In this approach, the problem of the model realizability does not arise. Thus, the QNSE-based RANS models present a viable alternative to the Reynolds stress closure models widely used in meteorological, oceanographic and engineering applications. The utility of the QNSE-based LES and RANS models and their performance are being further assessed in the ongoing theoretical and computational research.

Acknowledgements. This research has been partially supported by the US Army Research Office under Grant Nos. DAAD19-01-10816 and W911NF-05-1-0055, Israel Science Foundation under Grant No. 134/03, and the EU-funded project DAMOCLES. We are thankful to C. Edmisten of the Graphics Department of the College of Marine Science at USF for processing the figures.

Edited by: W.-G. Früh

Reviewed by: Two referees

\section{References}

Abarbanel, H., Holm, D., Marsden, J., and Ratiu, T.: Richardson number criterion for the nonlinear stability of three-dimensional stratified flow, Phys. Rev. Lett., 52, 2352-2355, 1984.

Andreas, E., Fairall, C., Guest, P., and Persson, P.: An overview of the SHEBA atmospheric surface flux programm, pp. 550-555, Amer. Meteorol. Soc., Dallas, TX, 1999.

Balsley, B., Frehlich, R., Jensen, M., and Meillier, Y.: Extreme gradients in the nocturnal boundary layer: Structure, evolution, and potential causes, J. Atmos. Sci., 60, 2496-2508, 2003.

Carnevale, G., Briscolini, M., and Orlandi, P.: Buoyancy- to inertial-range transition in forced stratified turbulence, J. Fluid Mech., 427, 205-239, 2001.
Curry, J., Rossow, W., Randall, D., and Schramm, J.: Overview of Arctic cloud and radiation characteristics, J. Climate, 9, 17311764, 1996.

Detering, H. and Etling, D.: Application of the $E-\epsilon$ model to the atmospheric boundary layer, Bound.-Layer Meteor., 33, 113$133,1985$.

Gargett, A., Hendricks, P., Sanford, T., Osborn, T., and Williams III, A.: A composite spectrum of vertical shear in the upper ocean, J. Phys. Oceanogr., 11, 1258-1271, 1981.

Howard, L.: Note on a paper of John W. Miles, J. Fluid Mech., 10, 509-512, 1961.

Kondo, J., Kanechika, O., and Yasuda, N.: Heat and momentum transfer under strong stability in the atmospheric surface layer, J. Atmos. Sci., 35, 1012-1021, 1978.

Kosovic, B. and Curry, J.: A large eddy simulation study of a quasisteady, stably stratified atmospheric boundary layer, J. Atmos. Sci., 57, 1052-1068, 2000.

Mack, S. and Schoeberlein, H.: Richardson number and ocean mixing: Towed chain observations, J. Phys. Oceanogr., 34, 736-754, 2004.

Mahalov, A., Nicolaenko, B., Tse, K., and Joseph, B.: Eddy mixing in jet-stream turbulence under stronger stratification, Geophys. Res. Lett., 31, doi:10.1029/2004GL021055, 2004.

McComb, W.: The Physics of Fluid Turbulence, Oxford University Press, 1991.

McComb, W.: Theory of turbulence, Rep. Progr. Phys., 58, 11171205, 1995.

Mellor, G. and Yamada, T.: Development of a turbulence closure model for geophysical fluid problems, Rev. Geophys. Space Phys., 20, 851-875, 1982.

Miles, J.: On the stability of heterogeneous shear flows, J. Fluid Mech., 10, 496-508, 1961.

Monti, P., Fernando, H. J. S., Princevac, M., Chan, W. C., Kowalewski, T. A., and Pardyjak, E. R.: Observations of Flow and Turbulence in the Nocturnal Boundary Layer over a Slope, J. Atmos. Sci., 59, 2513-2534, 2002.

Noh, Y., Kang, Y., Matsuura, T., and Iizuka, S.: Effect of the Prandtl number in the parameterization of vertical mixing in an OGCM of the tropical Pacific, Geophys. Res. Lett., 32, L23609, doi:10.1029/2005GL024540, 2005.

Perov, V. and Gollvik, S.: A 1-D test of a non-local $K-\epsilon$ boundary layer scheme for a NWP model resolution, HIRLAM Technical Report, 25, 1996.

Perov, V., Zilitinkevich, S., and Ivarsson, K.-I.: Implementation of new parameterisation of the surface turbulent fluxes for stable stratification in the 3-D HIRLAM, HIRLAM Newsletter, 37, 6066, 2001.

Poulos, G., Blumen, W., Fritts, D., Lundquist, J., Sun, J., Burns, S., Nappo, C., Banta, R., Newsom, R., Cuxart, J., Terradellas, E., Balsley, B., and Jensen, M.: CASES-99: A comprehensive investigation of the stable nocturnal boundary layer, Bull. Amer. Meteor. Soc., 83, 555-581, 2002.

Strang, E. and Fernando, H. J. S.: Vertical mixing and transports through a stratified shear layer, J. Phys. Oceanogr., 31, 20262048, 2001.

Sukoriansky, S., Galperin, B., and Perov, V.: Application of a new spectral theory of stably stratified turbulence to atmospheric boundary layers over sea ice, Bound.-Layer Meteor., 117, 231257, 2005a.

Sukoriansky, S., Galperin, B., and Staroselsky, I.: A quasinormal scale elimination model of turbulent flows with stable stratification, Phys. Fluids, 17, 085 107-1-28, 2005b. 
Uttal, T., Curry, J., McPhee, M., Perovich, D., Moritz, R., Maslanik, J., Guest, P., Stern, H., Moore, J., Turenne, R., Serreze, A. M., Wylie, D., Persson, O., Paulson, C., Halle, C., Morison, J., Wheeler, P., Makshtas, A., Welch, H., Shupe, M., Intrieri, J., Stamnes, K., Lindsey, R., Pinkel, R., Pegau, W., Stanton, T., and Grenfeld, T.: Surface heat budget of the Arctic Ocean, Bull. Amer. Met. Soc., 83, 255-276, 2002.
Yagüe, C. and Cano, J.: The influence of stratification on heat and momentum transfer in Antarctica, Bound.-Layer Meteor., 69, 123-136, 1994.

Yagüe, C. and Redondo, J.: A case study of turbulent parameters during the Antarctic winter, Antarctic Sci., 7, 421-433, 1995.

Yagüe, C., Maqueda, G., and Rees, J.: Characteristics of turbulence in the lower atmosphere at Halley IV station, Antarctica, Dyn. Atmos. Oceans, 34, 205-223, 2001. 